\title{
Keeping Up Electron Microscopy Diagnostic Skills in a PCR World
}

\section{Craig Radi}

Wisconsin Veterinary Diagnostic Laboratory, 445 Easterday Lane, Madison, WI 53706

craig.radi@wvdl.wisc.edu

\section{Introduction}

With the refinement of real-time polymerase chain reaction (RTPCR) techniques $[1,2]$ in diagnostic laboratories, negative stain [3] imaging of specimens for virus identification is on the decline in some electron microscopy (EM) diagnostic laboratories. Our lab went from over 2,000 negatively stained samples per year to less than 100. Most of these remaining samples were fecal content and intestine or intestinal content looking for enteric-type viruses because enteric diseases claim many livestock in the farm communities here in Wisconsin. This dramatic decrease in negatively stained samples can have a deleterious effect on keeping one's eye keen for spotting unknown pathogens in specimens received for transmission electron microscopy (TEM) examination.

In the last round of proficiency samples sent out by Berlin's Robert Koch Institute (RKI), samples were sent to only 92 laboratories, down from the previous 105 laboratories. This was in part because some labs never responded at all to the samples, stopped diagnostic EM altogether, or shut down once their scientists retired. Even in the recent round only 79 of those 92 labs sent back their findings. That sounds a bit disturbing to me in that we as diagnosticians are not keeping up with our abilities as microscopists.

Even though samples for TEM diagnostics have decreased at our lab, we still do a fair amount of sectioning for our pathology department and looking for unknown viruses that present themselves in tissue cell culture. Polymerase chain reaction techniques are limited to the availability of primers for a specific virus. A primer is a strand of nucleic acid that serves as a starting point for DNA synthesis. Thus, RTPCR is understandably more sensitive than EM because RTPCR has the ability to take as few as a single virus particle and amplify it into many more. However, the average time for a completed PCR analysis is still more than 2 hours, providing the run works and the controls pass validation when analyzed.

We had two cases a while back where some lesions from two birds were given not only to the molecular group, but to me as well to look for a potential pox virus. While they were still extracting the nucleic material from the specimen, I ground up a small piece of the lesion, added a few drops of $2 \%$ Phosphotungstic acid (PTA), applied a drop to a formvar carbon-coated grid, and within 10 minutes or so, I was imaging the pox virus that was in the sample. I brought back images to share while the extraction for RTPCR was still in process. The RTPCR test was a multiplex primer that could detect more than one type of avian pox virus. The results came back negative, because the primers were not able to pick up that particular type of avian pox virus. This was good because we recognized the need to alter the primer to include this virus, and it is now part of that RTPCR panel. Because EM does not require reagents specific to a virus or unknown agent, it can provide another window into the diagnostic process. It is important to use all the tools at your disposal to provide the best diagnostic service to your clients. That is why it is important for technologists to maintain a trained eye for recognizing viruses. This article describes a Round Robin program that can challenge labs to maintain their skills in virus identification.

\section{Training Program}

In 2007, the Centers for Disease Control (CDC) and Duke University partnered in a program for TEM labs in "Bioterrorism Training." Hosts Cynthia Goldsmith from the CDC and Sara Miller from the Department of Pathology at Duke saw a need for EM labs to learn techniques for identifying unknown agents that may be present in submitted specimens. The labs present for the course would be satellite laboratories that, in the event of a smallpox outbreak, for instance, could be helpful in identifying outbreaks in their area. Our lab in Madison actually has a plan in place with the local health department and the local hospitals in which we have set up protocols for responding to unusual outbreaks or lesions. The plan is periodically reviewed, and we included a simulation, which covered how, from the hospital to our lab, a specimen would be handled, shipped, and diagnosed. During these bioterrorism training sessions at the CDC, it was apparent that a number of labs did not have access to a variety of viruses or have the proper experience and techniques for identifying them. I suggested starting a program to send prepared grids of virus unknowns to the participating labs that attended the training program.

\section{Preliminary Round Robin}

Originally, a grid box was created with samples from the Wisconsin Veterinary Diagnostic Laboratory (WVDL), Duke University, and the CDC. Each laboratory prepared six copies of specimens containing a particular virus. These specimens included negative stained viruses using PTA and urynal acetate/lead citrate stained sections. The grids were assembled into one box that was mailed to the first lab, on a list of labs, that would observe, photograph, and diagnose. Participants would write their observations, take images of what they saw, and email their identification to me to receive the result key for that round. The box was then shipped to the next lab on the list. Average turnaround time was six to eight weeks. This procedure worked fairly well, but the grids were ripped or torn 


\section{Heat Up Your In-situ EBSD Work NEW Murano Heating Stage}
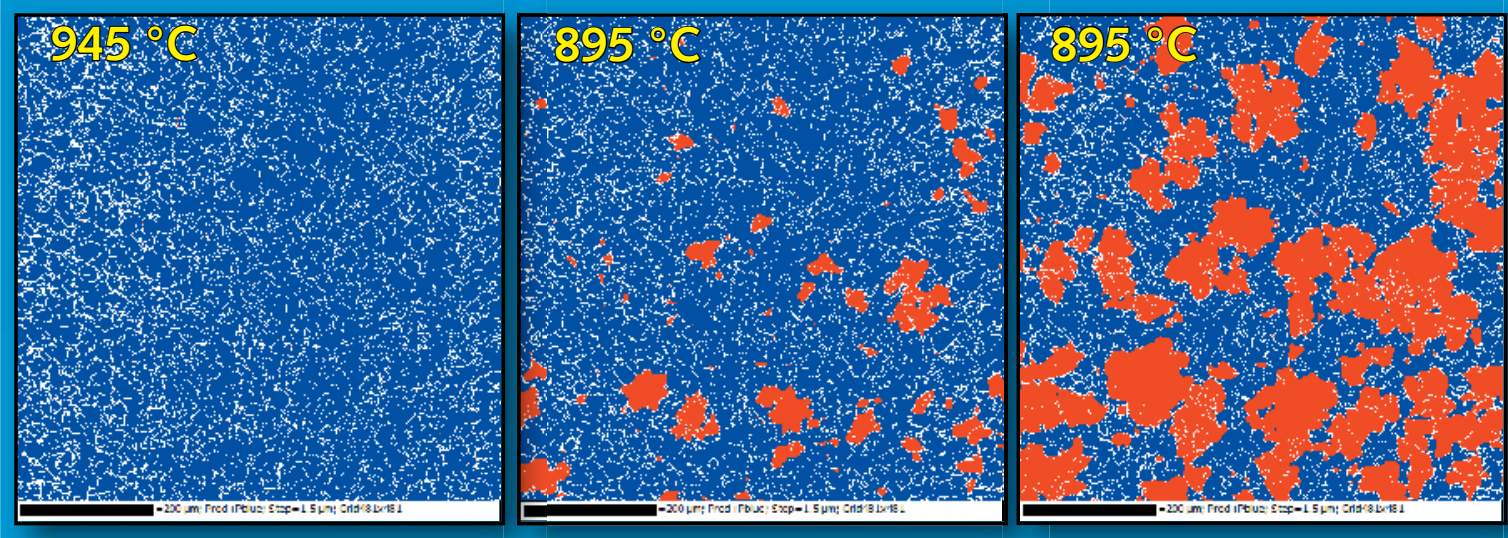

$880^{\circ} \mathrm{C}$

Murano Heating Stage delivers in-situ specimen characterization for your SEM

- Compatible with geometry constraints of EBSD / FIB /SED applications

- Study real time recrystallization and phase transformations from ambient to $950{ }^{\circ} \mathrm{C}$

- Removable platform for multiple specimens storage and mounting

- Optional gas injection to aid catalysis

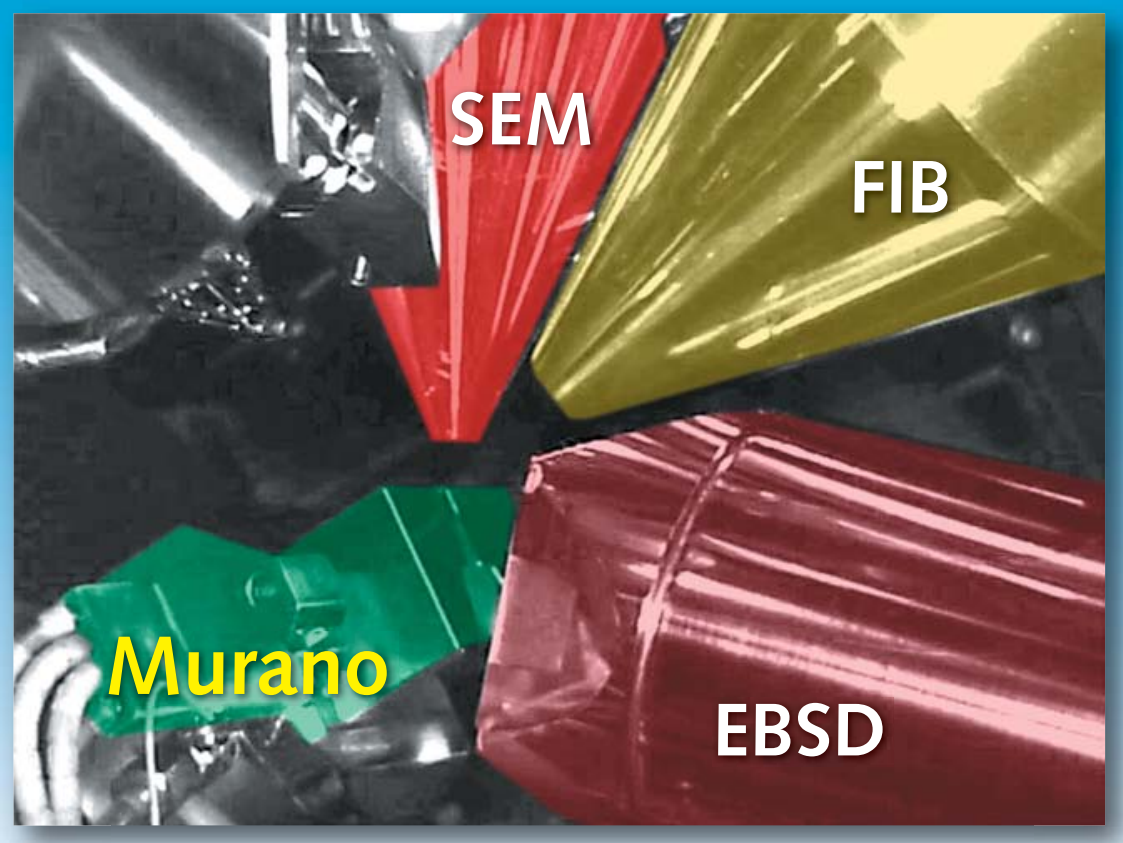

Top Images: EBSD phase maps showing austenite to ferrite transformation from $945{ }^{\circ} \mathrm{C}$ to $880{ }^{\circ} \mathrm{C}$, austenite is blue (dark) and ferrite is red (light). Measurements were taken using a single low carbon steel specimen heated to $945^{\circ} \mathrm{C}$ then cooled at $1{ }^{\circ} \mathrm{C}$ per minute until the start of phase transformation was observed. Once transformation started, temperature was held to observe development of the phase change in individual grains before cooling resumed. Data courtesy of Dr. Singh Ubhi from Oxford Instruments. Bottom Image: SEM chamber view with EBSD, FIB, SED.

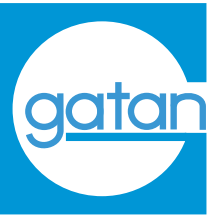

\section{Murano Heating Stage $950{ }^{\circ} \mathrm{C}$ In-situ Heating Stage for SEM FIB EBSD}

www.gatan.com 


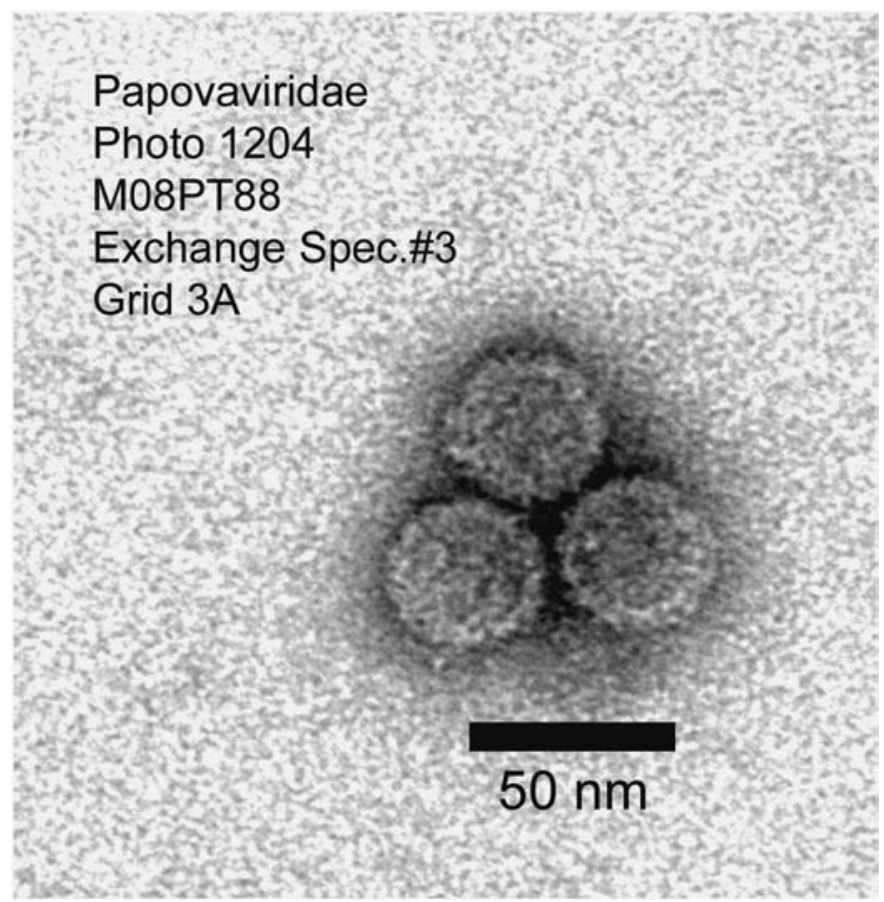

Figure 1: Papovaviridae. Image from the first year's session of prepared grids. Image by Margaret Casey.

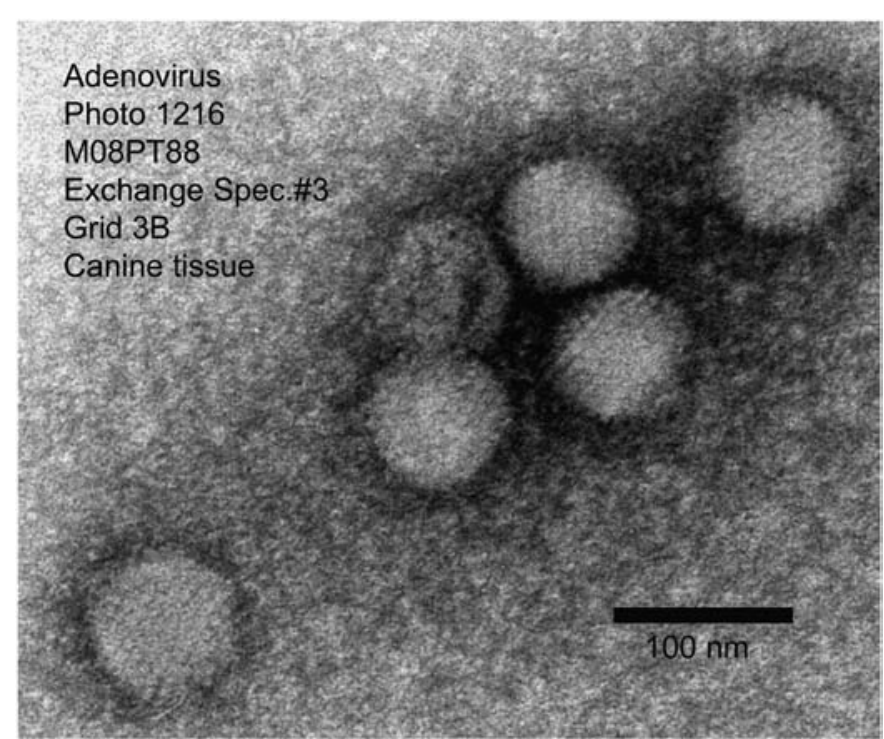

Figure 2: Adenovirus. Image from the first year's session of prepared grids. Image by Margaret Casey.

by time they got to the last lab. Figures 1 and 2 show examples of the premade grids that were sent to the labs for examination.

To address the poor integrity of the prepped grids, which resulted from the specimens being shipped and shared with multiple laboratories, WVDL propagated the viruses in tissue cell culture using bovine turbinate cells, spun them down at 100,000 RPM to obtain a more pure supernatant with a high load of viral particles, inactivated the viral supernatant in $2 \%$ paraformaldehyde, and shipped $1 \mathrm{ml}$ of the fixed samples to participating laboratories. This technique helped in two ways: (a) there was less damage to the grids and sections and (b) each lab had the opportunity to prepare and evaluate its

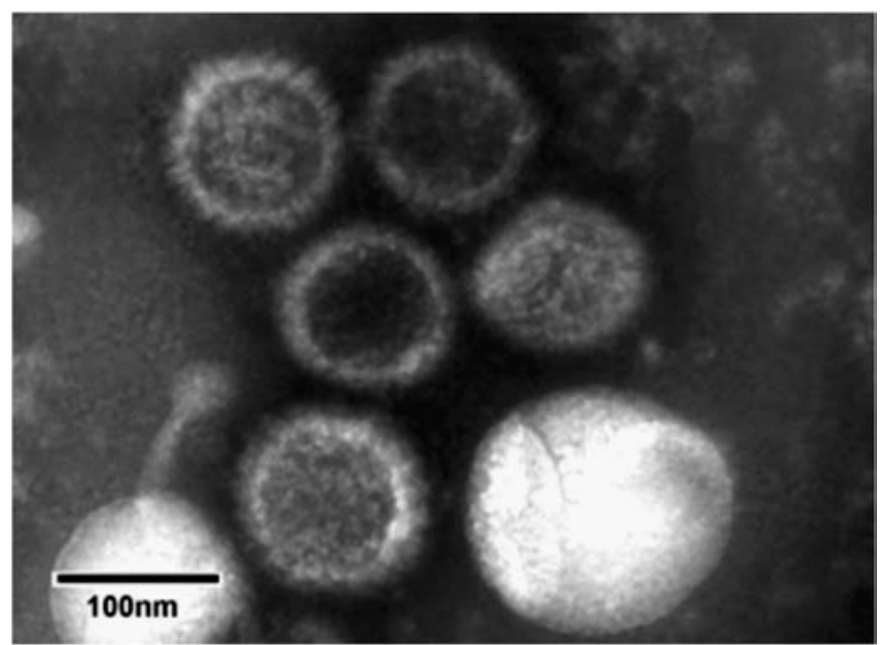

Figure 3: Image of a Herpesvirus on grids prepared by a participating lab. Image by Sara Miller.

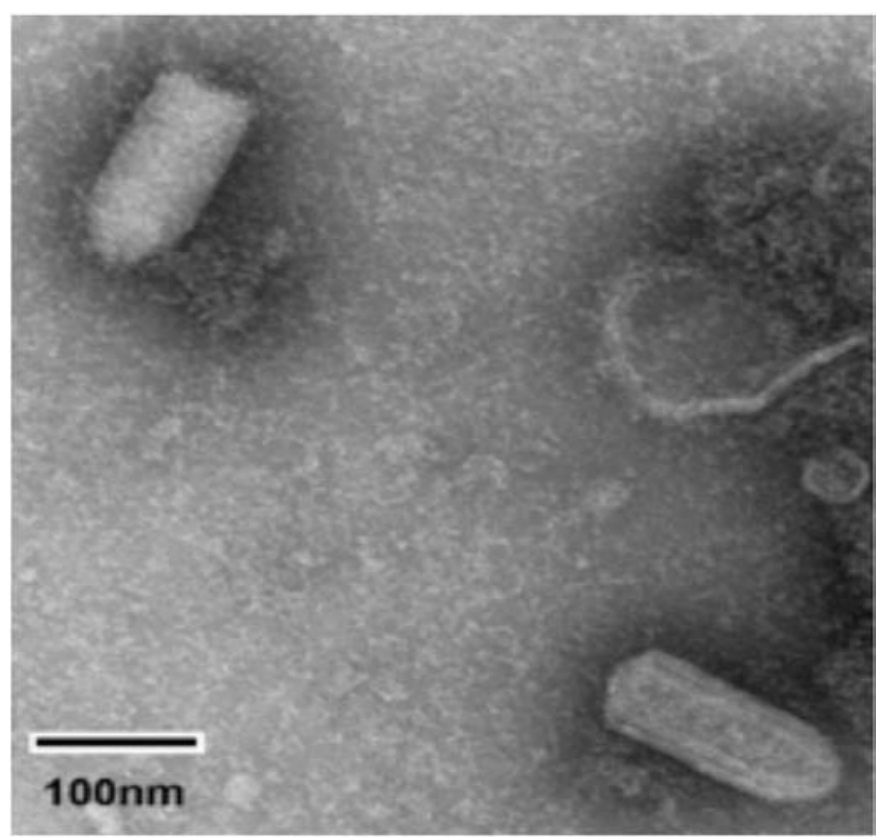

Figure 4: Image of a Rhabdovirus on grids prepared by a participating lab. Image by Margaret Casey.

own preparations, as well as make the diagnoses. Figures 3 and 4 show the isolates that were prepped by each lab and imaged. Participants were encouraged to try new techniques in staining and concentrating the virus onto the grid. Some of those techniques included using PTA, urynal acetate, and ammonium molybdate for staining and using poly-L-Lysine, agar diffusion, and ultra centrifugation for virus concentration on grids. This is the procedure we have followed for the last two years, and it has proven to be successful. For some of the labs, these are the only proficiency samples they receive all year.

An additional seven labs joined the program in 2011, and eight more signed up in 2012, for a total of 18 laboratories now participating in the Wisconsin EM Round Robin Program. The labs that have responded show great expertise in their abilities to prepare and observe unknown viruses [4] in a tissue culture 
Table 1: Recent results for the Wisconsin EM Round Robin Program

\begin{tabular}{|c|c|c|c|c|c|c|}
\hline Year & Agent & Matrix & $\begin{array}{c}\text { Participating } \\
\text { Labs }\end{array}$ & $\begin{array}{c}\text { Responding } \\
\text { Labs }\end{array}$ & $\begin{array}{c}\text { Confirmed } \\
\text { Virus ID }\end{array}$ & $\begin{array}{c}\text { Missed } \\
\text { Identification }\end{array}$ \\
\hline 2011 & Rhabdovirus & Cell Isolate & 10 & 6 & 6 & 0 \\
\hline 2012 & Herpesvirus & Cell Isolate & 18 & 13 & 12 & 1 Reovirus \\
\hline 2013 & Flavivirus & Cell Isolate & 18 & 10 & 10 & 0 \\
\hline
\end{tabular}

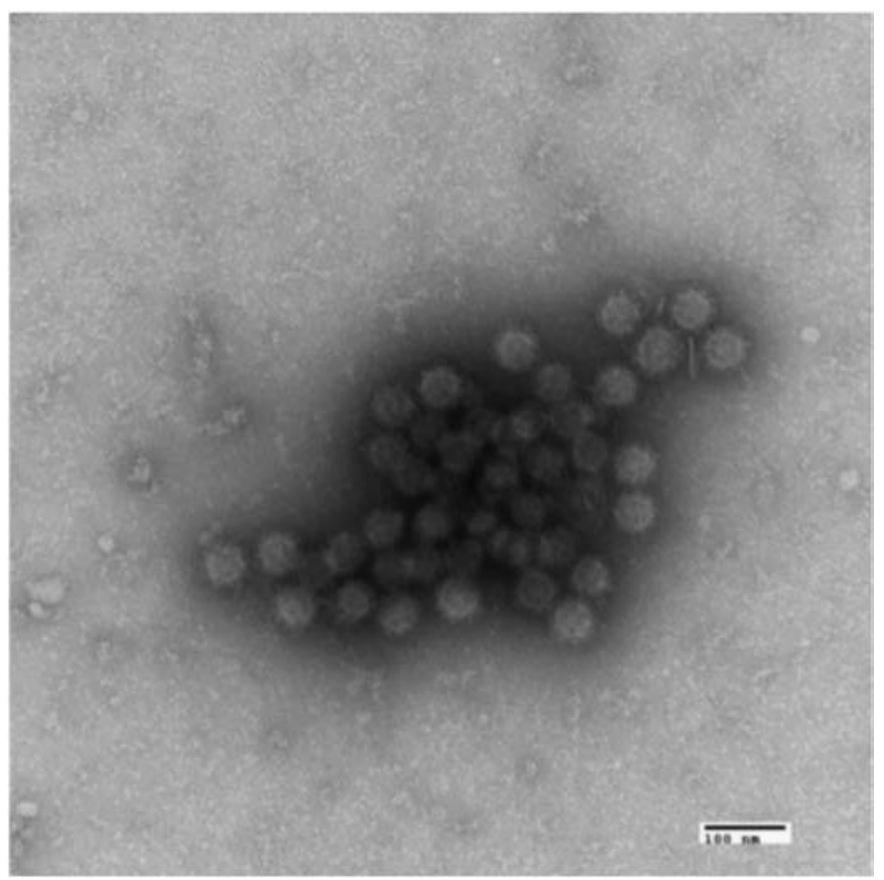

Figure 5: Image of a Flavivirus on grids prepared by a participating lab. Image by Cynthia Goldsmith.

isolate. I plan on sending out sectioned material for the labs to stain and observe so they can have experience preparing those types of samples as well.

\section{Results}

Table 1 shows data for the program over two years when the samples were prepared by the laboratories themselves. Figures 3, 4, and 5 show examples of images and preparation quality. Only one respondent lab had misidentified a virus. The labs that did not respond had problems with their microscope, did not have time to prepare samples, or had stopped providing EM services.

\section{Discussion}

There is another external quality assessment program that sends out samples for identifying viruses. This is offered by Lars Moeller of the Robert Koch Institute in Berlin. They send out six samples annually to applicants and send a certification of results that EM labs can use for their quality control programs. While this is a great program, some laboratories have problems with receiving samples from a foreign country. Our program helps fill in that gap for EM labs in the United States and Canada.

\section{Conclusion}

While the real-time polymerase chain reaction technique is becoming the standard in many diagnostic tests, it can miss unsuspected pathogens if the appropriate primers are not chosen or are not available. One should make use of all the tools in the diagnostic arsenal, including EM, when it comes to detecting unknown viruses in samples. Our round robin program not only offers samples for labs to prepare and observe, but some of the most respected scientists in the biological EM field are available for questions concerning technique and interpretation.

Author's note: If your laboratory is interested in participating in the next round robin program, please contact the author for more information.

\section{References}

[1] KT Kurth, T Hsu, ER Snook, EL Thacker, BJ Thacker, and FC Minion, J Vet Diagn Invest 14(6) 2002) 463-69.

[2] YQ Guo, DD Li, and ZJ Duan, Bing Du Xue Bao 29(6) (2013) 651-54.

[3] MA Hayat and SE Miller, Negative Staining, McGraw-Hill, New York, 1990.

[4] JJ Bozzola and LD Russell, Electron Microscopy: Principles and Techniques for Biologists, Jones and Bartlett Publishing, Sudbury, MA, 1998.

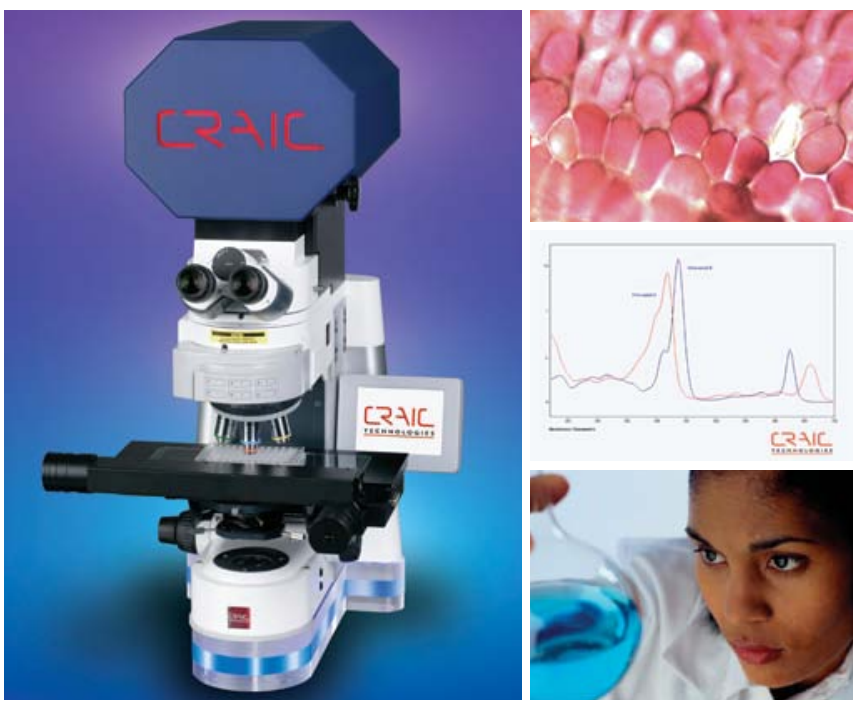

\section{SPECTROSCOPY OF MICROSCOPIC SAMPLES}

CRAIC Technologies UV-visible-NIR microscopes and microspectrophotometers are used for imaging and spectral analysis of sub-micron sized features with absorbance, reflectance, fluorescence, emission and polarized illumination. Capabilities include film thickness measurements, colorimetry and high resolution imaging in the UV, visible and NIR regions. Rapid \& accurate spectra \& images of microscopic samples: The Perfect Vision for Science ${ }^{\mathrm{TM}}$.

For more information, call 877.UV.CRAIC or visit our website at www.microspectra.com @2011 CRAIC Technologies, Inc. San Dimas, California (USA). 